\title{
Revolting Rhymes de Roald Dahl et Quentin Blake. Entre tradition et nouvelle jeunesse, ou les dents du conte retrouvées
}

\section{Florence Casulli}

\section{(2) OpenEdition \\ Journals}

Édition électronique

URL : https://journals.openedition.org/edl/1634

DOI : $10.4000 /$ edl. 1634

ISSN : 2296-5084

Éditeur

Université de Lausanne

\section{Édition imprimée}

Date de publication : 15 septembre 2019

Pagination : 167-188

ISBN : 978-2-940331-71-0

ISSN : 0014-2026

\section{Référence électronique}

Florence Casulli, «Revolting Rhymes de Roald Dahl et Quentin Blake. Entre tradition et nouvelle jeunesse, ou les dents du conte retrouvées », Études de lettres [En ligne], 310 | 2019, mis en ligne le 15 septembre 2021, consulté le 21 septembre 2021. URL : http://journals.openedition.org/edl/1634 ; DOI : https://doi.org/10.4000/edl.1634

Ce document a été généré automatiquement le 21 septembre 2021.

(c) Études de lettres 


\title{
Revolting Rhymes de Roald Dahl et Quentin Blake. Entre tradition et nouvelle jeunesse, ou les dents du conte retrouvées
}

\author{
Florence Casulli
}

1 Revolting Rhymes (1982) est un recueil de six contes classiques détournés par Roald Dahl (1916-1990) et illustrés par Quentin Blake (1932). Dans ces nouvelles versions mises en vers principalement destinées aux enfants, l'auteur procède à des «mises à jour » de l'univers du conte et de ses codes avec humour et une pointe de satire. Dahl propose en effet à ses lecteurs jeunes et moins jeunes une relecture iconoclaste des contes traditionnels qui, loin des versions édulcorées ou sentimentales popularisées par Disney, revisite les sources textuelles de Perrault et Grimm et leur réception à partir d'un point de vue contemporain. Revolting Rhymes surprend, amuse et provoque en faisant voler la morale traditionnelle en éclats, tout en la renouvelant subtilement pour un lecteur d'aujourd'hui ${ }^{2}$. L'œuvre s'élabore ainsi en " contrepoint» des versions bien connues de "Cinderella», "Jack and the Beanstalk», "Snow-White and the Seven Dwarfs ", « Goldilocks and the Three Bears ", « Little Red Riding Hood and the Wolf » et « The Three Little Pigs ", et nous incite à mesurer l'écart entre passé et présent.

Dahl transpose les histoires familières dans le contexte social et culturel des années 1980. Le ton irrévérencieux, l'humour féroce, les références au monde moderne, le renversement des rôles où les victimes deviennent parfois bourreaux, créent un effet jubilatoire de monde à l'envers pour le jeune lecteur et plus encore pour le lecteur adulte dans la mesure où « le plaisir de la lecture surgit des écarts entre le souvenir de l'histoire familière et la surprise de sa réinterprétation ou de son détournement, qui déjoue les attentes du lecteur et renouvelle le sens du conte $»^{3}$. Désormais inséparable des livres de Dahl, le trait rapide de Quentin Blake est doté d'un humour plus léger, burlesque et espiègle qui atténue la cruauté du récit tout en mettant en évidence l'actualité toujours renouvelée du conte. 
Revolting Rhymes est une réécriture hybride formant un "iconotexte ${ }^{4}$ qui s'inspire librement de la réception éditoriale et iconographique des contes de Perrault et Grimm en Angleterre et aux États-Unis, bien que l'imaginaire du conte soit désormais globalisé5. Il semble néanmoins que Dahl et Blake inscrivent leur démarche dans une tradition satirique du genre qui va des caricatures de George Cruikshank pour la première traduction-adaptation anglaise des contes de Grimm, à l'humour drolatique et grotesque des dessins animés de Tex Avery en passant par la tradition anglaise des Mother Goose Rhymes. Se focalisant principalement sur trois contes du recueil, "Cinderella ", «Little Red Riding Hood and the Wolf » et " The Three Little Pigs », cet article propose que l'iconotexte de Revolting Rhymes revisite cette tradition textuelle, visuelle et culturelle des contes afin de leur donner une nouvelle jeunesse et un nouveau mordant.

\section{1. « Le coup de jeune » des réécritures de Dahl}

Comme annoncé dans le titre, Revolting Rhymes rassemble des réécritures révoltantes de contes traditionnels destinées tout à la fois à provoquer le lecteur par leur contenu violent, peu ragoûtant, voire immoral, tout en jouant sur le côté jubilatoire de la transgression des codes et des normes du genre, et bien sûr de sa dimension pédagogique et édifiante. Il met en évidence l'aspect parodique et iconoclaste du recueil qui renverse les rôles (volvere), retourne les situations et propose des dénouements surprenants à la façon des nursery rhymes, ces comptines versifiées pour les enfants souvent publiées dans les mêmes éditions que les contes en Angleterre ${ }^{6}$. En revisitant les contes qui « ont longtemps servi à instruire et même à communiquer des vérités amères sous une forme plaisante et " puérile " " ${ }^{7}$ sur le mode des nursery rhymes au contenu souvent dérangeant, transgressif, voire subversif, Dahl provoque, amuse et moralise à sa façon ${ }^{8}$. Il donne ainsi une nouvelle jeunesse à cette tradition en mêlant drôlerie enfantine, cruauté et satire sociale à travers la figure du Petit Chaperon rouge, parmi d'autres.

5 Dahl réécrit les contes en rimes plates (typiques de la poésie comique, limericks et nonsense verse) en digne héritier d'Edward Lear et de James Thurber. Il renouvelle et réactualise cette tradition par l'utilisation de mots argotiques, d'onomatopées et de formules fantaisistes comme « Mind your own bizz » (p. 11) ou « We're stony broke» (p. 13) et opte pour un cadre à la fois moderne, familier et populaire : le bal de Cendrillon devient une fête «disco" (p. 6) et le miroir magique de Blanche-Neige permet de gagner une fortune aux courses hippiques. Le recueil s'ouvre sur "Cinderella", qui débute par cet avertissement au lecteur :

I guess you think you know this story.

You don't. The real one's much more gory.

The phoney one, the one you know,

Was cooked up years and years ago,

And made to sound all soft and sappy

Just to keep the children happy (p. 5).

6 Se démarquant des textes expurgés et des adaptations édulcorées à destination des enfants, le conteur se réclame d'une tradition jugée plus authentique associée au folklore et à la culture populaire - en un mot, plus sanglante ("gory» rimant ironiquement avec "sappy " et "happy »). Comme le remarquent les éditrices du premier ouvrage académique qui lui est consacré, «Dahl demonstrates his awareness 
that stories are put to use [...] for particular purposes and that the stories typically read to children are de-fanged $»^{9}$. Il restitue donc la cruauté des contes à l'image de celui de «Jack and the Beanstalk» et de sa célèbre formulette «FEE FI FO FUM/I SMELL THE BLOOD OF AN ENGLISHMAN ! (p. 16), et souligne la portée satirique, critique et morale $\mathrm{du}$ genre $^{10}$. Sous couvert de provocation ou d'espièglerie, Dahl propose ainsi une petite leçon invitant le lecteur à revenir à la tradition orale afin de redécouvrir les aspects méconnus et subversifs d'un folklore féroce et sans tabou qui prend le parti des enfants contre les ogres et les méchants, mais sans niaiserie ni sentimentalisme.

$7 \quad$ Les réécritures de Dahl soulignent par exemple la dimension métatextuelle des recueils et leur contexte de production et réception, en se démarquant des idées reçues sur le conte comme histoire naïve située hors du temps et $d u$ monde ${ }^{11}$. Par exemple, le conteur attire l'attention tout à la fois sur les filiations et les détournements qu'il fait subir au texte $d u$ "Petit Chaperon rouge $»^{12}$. Lorsque cette dernière fait des compliments au loup qui porte le manteau de fourrure de sa grand-mère, celui-ci s'exclame :

"That's wrong!" cried Wolf. " Have you forgot

To tell me what BIG TEETH I've got?" (p. 40)

8 Comme dans le Red Hot Riding Hood (1943) de Tex Avery où les personnages sont lassés de jouer toujours les mêmes rôles, les parodies de Dahl mettent en évidence le fonds commun tout en renouvelant le sens et la portée critique des récits familiers. Comme le résume bien Catherine d'Humières, Dahl « remet en cause le genre du conte par l'absence de merveilleux, les anachronismes, lui donne un "coup de jeune " et met en abyme le travail des écrivains ${ }^{13}$. L'auteur se plait à redécouvrir derrière les versions édulcorées l'ironie ou les détails macabres afin de se moquer des clichés associés au genre. Cette charge satirique se retrouve notamment dans « Little Red Riding Hood and the Wolf » et "The Three Little Pigs », deux contes réunis par le personnage du loup et, de façon plus inattendue, du «Petit Chaperon rouge » qui retourne la situation à son avantage. Dans la première réécriture, la fillette n'est plus l'héroïne naïve et ignorante des dangers de ce monde qui se fait dévorer par le loup (chez Perrault), ou qui n'échappe à celui-ci que grâce à un chasseur (chez Grimm): indépendante, entreprenante et coquette dans un monde où "l'homme est un loup pour l'homme " (femmes comprises), c'est elle qui, en "brave, active, "modern" girl ${ }^{14}$, déjoue les ruses du loup, le tue et ... s'en fait un manteau de fourrure :

She said, ' Hello, and do please note

'My lovely furry WOLFSKIN COAT' (p. 40).

La modification des noms des deux personnages par Dahl souligne d'ailleurs le fait que les rôles sont inversés. Comme le note Sandra Beckett, "the diminutive "Wolfie" immediately reduces radically the intimidating nature of the wolf, whereas the more formal "Little Miss Red Riding Hood" increases the status of the heroine $»^{15}$. Ainsi, alors même que la revanche de l'héroïne sur le loup prédateur est jubilatoire pour quiconque s'identifie à la petite fille, Dahl complique la morale du conte en dénonçant aussitôt des formes très contemporaines de prédation féminine ${ }^{16}$. Beckett insiste d'ailleurs sur le fait que le meurtre du loup est un acte prémédité qui confirme la nature prédatrice et sans scrupules de la fillette: "This is clearly not a case of selfdefense, but a premeditated murder devised by an unscrupulous little girl who has no qualms whatsoever about wearing fur coats $»^{17}$. 
10 Dans « The Three Little Pigs ", l'un des trois petits cochons sollicite l'aide de la fillette pour échapper au loup. Mal lui en prend, car l'héroïne fashion victim (et material girl) est dépourvue de tout sens moral: elle n'hésite pas à tuer son ami pour en faire un «PIGSKIN TRAVELLING CASE» (p. 47). Dahl réactualise ainsi ses sources en commentateur avisé d'une époque complexe où les proies d'hier se changent parfois en prédateurs à l'ère du postféminisme.

\section{Les illustrations de Blake : clins d'œil et sourires (avec ou sans les dents)}

11 Si Revolting Rhymes revisite délibérément les versions des contes popularisées par Perrault et Grimm, les illustrations nouent un dialogue complexe avec le texte, mais aussi avec la tradition illustrée des contes. Les archives de l'auteur témoignent de son intérêt pour l'illustration, qui constitue selon lui une condition sine qua non pour créer un véritable livre pour enfants. Ainsi, dans la correspondance qu'il a entretenue avec l'un de ses éditeurs, Tom Maschler, Dahl déclare qu'il ne conçoit pas d'écrire un livre pour enfants qui ne soit richement illustré : « There is no way in which I will permit a major children's book of mine to be published with only twelve illustrations ${ }^{18}$. Dès les premiers brouillons de ses ouvrages pour la jeunesse, Dahl n'hésite pas à semer, dans les marges de ses récits, des annotations destinés aux illustrateurs. L'un des brouillons de James and the Giant Peach est, par exemple, parsemé d'indications comme «small picture. Sad boy, aged about seven, ragged clothes ${ }^{19}$. Dans l'incipit de Charlie and the Chocolate Factory, le lecteur est directement invité à découvrir la famille Bucket comme on découvre un album photo, le texte et les illustrations se répondant mutuellement et rendant la lecture extrêmement dynamique.

12 La première collaboration entre Roald Dahl et Quentin Blake date de 1978 avec The Enormous Crocodile, le premier livre pour enfants que Dahl a conçu comme un véritable album (picturebook). Dans Revolting Rhymes, le texte « appelle » donc les images de Blake qui soulignent les temps forts du récit, illustrent des scènes non narrées, permettent de visualiser les personnages et les décors dans lesquels ils évoluent et infléchissent le ton et l'humour de certains épisodes ${ }^{20}$. Elles compensent ainsi le déficit descriptif du texte, tout en jouant un rôle essentiel dans la dimension humoristique et ironique du conte ${ }^{21}$. Privilégiant l'ellipse, le trait vif et l'allusion, l'illustrateur interprète et élabore des détails visuels tirés $d u$ texte ${ }^{22}$. Ainsi, Blake représente les méchantes sœurs de Cendrillon de façon stéréotypée, l'une grande et maigre au nez pointu et l'autre petite et corpulente au nez retroussé, à partir d'un seul élément textuel les qualifiant de laides : ce double portrait orne la table des matières, donnant en quelque sorte le ton $\mathrm{du}$ recueil. L'illustrateur joue sur les contrastes propres au conte qui associe souvent apparence et caractère, bien que la légèreté du trait et l'humour "cartoonesque " atténuent la charge satirique du texte.

De la même manière que Dahl joue avec l'intertextualité des contes, Blake propose une certaine intericonicité en faisant référence de façon plus ou moins délibérée à ses prédécesseurs, même si sa technique évoque surtout la culture populaire, la bande dessinée et les dessins animés. L'apparence qu'il donne à son Petit Chaperon rouge dans le médaillon (p. 38), par exemple, évoque le portrait de John Everett Millais en 1865. Comme chez Millais, on retrouve une fillette aux cheveux blond vénitien, vêtue d'une cape rouge vif et portant un panier de victuailles à sa grand-mère (fig. 1). Cependant, la 
candeur du modèle de Millais (nez retroussé, jeune âge, grands yeux ingénus et posture timide) cède le pas à la rouerie du personnage représenté par Blake, que vient confirmer le portrait en pied de la page 39 où l'enfant minaudant fait face au lecteur et, tout sourire, prend la pose avec coquetterie dans son nouveau manteau de fourrure.

$<$ Image en attente de droits>

Fig. 1 - John Everett Millais, « Little Red Riding Hood », 1865.

En réponse à Dahl, qui insiste sur la nouvelle personnalité de la fillette dont l'autonomie et le courage font bientôt place à l'opportunisme et à la vanité, Blake propose une version modernisée du "Petit Chaperon rouge» qui évolue d'une illustration à l'autre et déjoue une lecture manichéenne du conte. La fillette blonde de la couverture, apeurée, grandit en quelque sorte de page en page : elle apparaît bientôt seule, suggérant une certaine autonomie puis, dans l'illustration occupant toute la belle page, pose de façon presque aguicheuse au cœur de la forêt. Le lieu ne représente plus une menace puisque l'héroïne, souriante et confiante, est au centre de l'image et exhibe fièrement sa pelisse au lecteur. Sa cape rouge vif a disparu pour faire place à un manteau de fourrure imposant (hérissé de poils, en écho visuel aux branches des sapins mais aussi aux dents et aux griffes du loup) et des chaussures roses à talons hauts (fig. 2). La première illustration du conte (p. 37) évoque aussi lointainement le loup anthropomorphisé de Walter Crane (1875), déguisé en berger (une peau de mouton, comme dans la fable d'Ésope). Dans la version moderne et comique proposée par Blake, le loup porte un ensemble tailleur-jupe gris emprunté à la grand-mère, des chaussures à talons et un chapeau à fleurs : dans les deux illustrations, la longue gueule pleine de dents, l'œil jaune et rouge et la langue pendante dénotent la bestialité de l'animal, même si le travestissement du prédateur en vieille femme déjoue le tragique de la situation (fig. 3 et 4 ). Le contexte de production, transmission et réception des textes est mis en évidence sur la couverture de Revolting Rhymes où figure le loup emblématique avec, sur ses genoux, deux enfants terrorisés auxquels il raconte une histoire qui fait peur (peut-être «Le Petit Chaperon rouge » ou sa suite macabre) dans une mise en abyme du recueil (fig. 7). Dans le livre, les illustrations encadrées (rectangle ou médaillon) relèvent également de la métafictionnalitée ${ }^{23}$. Alors que cellesci rappellent les limites matérielles du support, les images sans bordure sur une page entière ou une double page "produisent cette impression de pouvoir se prolonger audelà de la double page $»^{24}$. En variant ainsi le format, le cadre, la taille et la disposition des illustrations (insérées parfois dans le texte), Blake met en évidence les procédés liés à la fabrication du livre et à sa réception, en lien avec la scène d'énonciation et de transmission des contes qui figure sur la couverture. Fidèle à la stratégie de Dahl consistant à mettre en avant le processus de création du texte dans un mille-feuille à la fois textuel, visuel et filmique (cinéma, dessins animés, etc.), Blake a recours à des procédés qui inscrivent les histoires dans un contexte, des formes de récit et des références culturelles contemporaines. La présence d'une télévision dans son illustration du loup s'habillant en grand-mère rappelle la double filiation - érudite et populaire - du genre, la télévision étant l'un des principaux instruments de médiation du conte au XXe siècle.

$<$ Image en attente de droits>

Fig. 2 - Quentin Blake, «Little Red Riding Hood and the Wolf », 1982. @ Quentin Blake 1983.

$<$ Image en attente de droits>

Fig. 3 - Walter Crane, «Little Red Riding Hood and the Wolf», 1875. 
$<$ Image en attente de droits>

Fig. 4 - Quentin Blake, «Little Red Riding Hood and the Wolf », 1982. @ Quentin Blake 1983.

Dans son ouvrage intitulé Words and Pictures (2000), Blake décrit sa méthode dite de la boîte lumineuse $\mathrm{e}^{25}$. Il explique aussi que les illustrations de Revolting Rhymes devaient initialement être en noir et blanc, un choix qu'il avait jugé justifié vu le caractère sombre et sarcastique des réécritures de Dahl. Or, l'éditeur lui demanda peu après de les réaliser en couleur, sans doute pour ne pas effaroucher un public enfantin. L'illustrateur dut alors adapter sa technique et aquarelliser ses dessins :

Then I remembered the tinted versions I had seen of nineteenth century prints lithographs by Daumier and Gavarni. Perhaps my drawings would also accept those pale washes of watercolour. I photocopied the illustrations on to more sheets of watercolour paper and experimented ${ }^{26}$.

Revolting Rhymes s'inscrit donc dans « une longue série d'éditions illustrées, selon une chronologie et des modalités de mise en images des contes $»^{27}$ associant le conte et l'image, en perpétuant l'esprit burlesque des illustrations réalisées par Cruikshank et ses successeurs.

\section{Revolting Rhymes et le topos détourné, ou comment le loup/lecteur dévore désormais les livres de contes}

Tous les livres pour la jeunesse de Roald Dahl empruntent à la tradition des contes de façon plus ou moins avouée, à commencer par ses plus grands succès comme James and the Giant Peach (1961), Charlie and The Chocolate Factory (1964) ou encore Matilda (1988). Selon David Rudd, Dahl se plaisait à adopter la posture du conteur qui amuse et divertit, intervenant pour commenter ses récits : « Known as a witty raconteur and storyteller (his own children being his primary audience), Dahl seems to carry this style into his writing, where we always have intrusive, opinionated narrators $»^{28}$. En particulier, la narration au présent, les nombreux déictiques, ou encore le ton familier évoquent des scènes de parole typiques du conte ${ }^{29}$. La mise en scène de la transmission des contes par la parole, le texte et l'image constitue par ailleurs un topos des livres de contes et comme le souligne David Rudd, «Dahl's own writing is powerful in conjuring up visual images $»^{30}$. Revolting Rhymes s'inscrit donc dans la double filiation d'une poétique de l'oralité et d'une riche tradition du livre illustré. Quentin Blake considère d'ailleurs que "le dessin est un langage à part, qui vous permet d'exprimer des choses que les mots ne peuvent pas toujours dire $»^{31}$. L'illustration de couverture met en évidence l'inscription générique des contes en détournant le fameux frontispice du recueil de Perrault par Clouzier, et plus encore celui par Doré avec le fauteuil, les enfants sur les genoux et les personnages de contes encadrés dans une mise en abyme du genre (fig. 5-7). À l'instar de ces frontispices, l'illustration de Blake figure «la mise en représentation de la narration de ces histoires, celle de leur énonciation ${ }^{32}$. Comme Louis Marin l'a bien montré, de nombreux contes ont en effet pour thème les effets de la parole, même si «la représentation visuelle de leur transmission orale est [aussi] un trait caractéristique des recueils $»^{33}$. Quentin Blake s'inspire de cette tradition en représentant le loup du "Petit Chaperon rouge » qui se substitue à la mère-grand conteuse d'histoires et assume dès lors le rôle d'une nouvelle "Ma Mère l'Oye ". L'humour réside non seulement dans le renversement des rôles, mais aussi dans la 
figure d'un loup dévorant désormais les histoires plutôt que les petits lecteurs apeurés, peut-être parce que loin d'être bien-pensantes les versions qu'en propose Dahl sont « croquignolettes »:

La couverture de Revolting Rhymes joue aussi sur l'inversion des rôles et la mise en abyme comique (dans la tradition de l'humour noir britannique). La grand-mère conteuse se transforme en loup vorace qui se lèche les babines à la lecture du conte de Perrault, dans une image qui capte admirablement l'aspect cruel et inquiétant des contes ${ }^{34}$.

$<$ Image en attente de droits>

Fig. 5 - Antoine Clouzier, Frontispice des Histoires ou contes du temps passé, 1697.

$<$ Image en attente de droits>

Fig. 6 - Gustave Doré, Frontispice des Histoires ou contes du temps passé, 1862.

$<$ Image en attente de droits>

Fig. 7 - Quentin Blake, couverture de Revolting Rhymes, 1982. (c) Quentin Blake 1983.

Si la version de Perrault fait la part belle au loup (qui dévore le Petit Chaperon rouge), celles de Dahl jouent elles aussi la provocation en déjouant les attentes du lecteur et les morales attendues, Blake soulignant ainsi l'aspect revolting des textes de Dahl où les rôles familiers s'échangent et se renversent. Si le loup, malgré son aspect menaçant, divertit les enfants et se régale désormais d'histoires plus que de chair humaine (en tout cas dans un premier temps), le danger est ailleurs : il faut se méfier des fausses évidences et particulièrement de ses anciennes victimes, comme le Petit Chaperon rouge (et, déjà, Cendrillon réclamant des cadeaux à sa marraine), désormais avide de biens de consommation et d'accessoires de mode. La malveillance initiale du loup est alors atténuée par l'attitude perverse de l'héroïne qui n'a pas de scrupules à tuer des animaux pour enrichir sa garde-robe ${ }^{35}$. Dahl souligne la satire sociale de Dahl en la représentant dans "The Three Little Pigs » se prélassant en peignoir sur un canapé et entourée d'objets contemporains qui symbolisent le culte de l'apparence et l'oisiveté : sèche-cheveux, téléphone et pile de magazines de mode. L'ère de la consommation de masse qui transforme les individus en prédateurs narcissiques et égoïstes est désormais la cible de la satire. C'est moins des loups dont il faut se méfier désormais, mais bel et bien des jeunes femmes qui ne reculent devant rien pour assouvir leurs désirs, Revolting Rhymes proposant ainsi un commentaire en demi-teinte des avancées féministes à l'ère du capitalisme triomphant.

Dahl aborde d'autres sujets de société avec une ironie mordante, en n'hésitant pas à remettre la violence des contes classiques au goût du jour, comme la version de "Cinderella » façon Grimm. Dans la version modernisée, les sœurs ne sont pas punies cruellement par l'oiseau vengeur qui leur crève les yeux à l'entrée et à la sortie de l'église : c'est le Prince qui, loin d'être charmant, se révèle être un tyran sanguinaire, capricieux et narcissique (une manière de Barbe bleue). Frustré de ne pas trouver de prétendantes à son goût, il leur coupe la tête. Dahl décrit la scène avec force détails macabres et grandguignolesques :

Her head went crashing to the ground.

It bounced a bit and rolled around.

In the kitchen, peeling spuds,

Cinderella heard the thuds [...] (p. 11).

Margaret Talbot relève toutefois que les illustrations de Blake atténuent cette violence par une touche comique: "The violent subtext of the book is often leavened by the 
delightful illustrations of Quentin Blake ${ }^{36}$, ce que Blake confirme : «I made sure there was no blood ; the head comes off like a doorknob that might be fitted on again late $»^{37}$.

Le traitement visuel de la scène évoque d'ailleurs un dessin de James Thurber, auteur d'un «The Little Girl and the Wolf» (1943) auquel Blake semble rendre hommage (fig. 8-9). Dans sa version de "Cinderella", le conteur nous rappelle que les sœurs de Cendrillon sont mauvaises et méritent donc en quelque sorte leur punition, invitant implicitement le lecteur à ne pas être choqué par le sort qui leur est réservé, mais l'illustration de Blake n'active pas le sens de dégoûtant, horrifiant et révulsant de revolting dans l'image. Il utilise à nouveau ce procédé dans « Little Red Riding Hood and the Wolf ». Au lieu d'illustrer la dévoration de la grand-mère, Blake représente l'aprèsdévoration de cette dernière plutôt que l'acte lui-même: "He ate her up in one big bite» (p. 36) ${ }^{38}$. Dans l'illustration, seuls quelques détails témoignent de la scène macabre : les lunettes de la grand-mère et son tricot sur le sol suggèrent la rapidité avec laquelle elle a été engloutie. À nouveau, aucune tache de sang n'est visible. Seule la couleur de la langue et les yeux injectés de sang du loup la suggèrent indirectement. Alors que Dahl n'hésite pas à décrire des scènes cruelles rappelant sur un mode drolatique les versions non expurgées des contes, Blake prend le parti d'«all[er] un peu plus loin que les simples mots [et en] y apport[ant] un petit supplément burlesque $»^{39}$.

$<$ Image en attente de droits>

Fig. 8 - Quantin Blake, «Cinderella », 1982. @ Quentin Blake 1983.

$<$ Image en attente de droits>

Fig. 9 - James Thurber, «Touché! », 1943.

\section{Conclusion}

Les réécritures de Dahl se situent donc dans la continuité de la tradition des contes en revisitant les versions classiques pour restituer et actualiser la portée sociale et critique du genre - son côté mordant, si l'on veut. Destiné à un double public composé de jeunes lecteurs et de lecteurs adultes, le livre offre une nouvelle jeunesse aux contes en les inscrivant dans un contexte contemporain, la charge satirique étant quelque peu atténuée dans les illustrations humoristiques, gaies et colorées de Blake. Ainsi, le jeu du texte et de l'image interpelle le lecteur non seulement dans sa dimension d'affect (revolting, mais aussi surprise, peur, rire, dégoût, etc.) mais il invite le lecteur adulte à prendre la mesure de la portée subversive des contes, leur côté grinçant et leur satire des mœurs à chaque époque, et leur donne ainsi une nouvelle jeunesse et actualité.

\section{BIBLIOGRAPHIE}

\section{Sources}

DAHL, Roald, Charlie and the Chocolate Factory, New-York, Puffin Books, 2007. 
-, James and the Giant Peach, New-York, Puffin Books, 2007.

-, Revolting Rhymes, New-York, Puffin Books, 2001.

-, The Enormous Crocodile, New-York, Puffin Books, 2009.

GRIMM, Jacob et Wilhelm, Contes, trad. par A. Guerne, Paris, Gallimard, 1976.

PERRAULT, Charles, Histoires ou contes du temps passé, présentation et introduction par Marc

Fumaroli, Paris, Flammarion, 2014.

\section{Travaux}

ALSTON, Ann, BUTLER, Catherine (eds), Roald Dahl, Basingstoke, Palgrave Macmillan, 2012.

AVERY, Tex, Red Hot Riding Hood, Metro-Goldwyn-Mayer et Loew's Incorporated, 1943.

BADER, Barbara, American Picturebooks from Noah's Ark to The Beast Within, New York, Macmillan, 1976.

BARDIN, Laurence, « Le texte et l'image », Communication et Langage, 26 (1975), p. 98-112.

BECKETT, Sandra, « Perverse, Pistol-Packing Riding Hoods », in Recycling Red Riding Hood, New York, Routledge, 2002, p. 124-129.

BLAKE, Quentin, Words and Pictures, London, Tate Publishing, 2000.

BURTON-HILL, Clemency, « The dark side of nursery rhymes », BBC Online, 11 juin 2015 (<http://

www.bbc.com/culture/story/20150610-the-dark-side-of-nursery-rhymes>).

Collectif, Roald Dahl, le géant de la littérature jeunesse, Paris, Gallimard jeunesse, 2016.

D'HUMIÈRES, Catherine (éd), D'un conte à l'autre, d'une génération à l'autre, Clermont-Ferrand, Presses universitaires Blaise Pascal, 2008.

DOLLERUP, Cay, Tales and Translation. The Grimm Tales from Pan-Germanic Narratives to Shared International Fairytales, Amsterdam/Philadelphy, John Benjamins Publishing Company, 1999.

EVRARD, Franck, L'humour, Paris, Hachette, 1996.

GENETTE, Gérard, Palimpsestes : la littérature au second degré, Paris, Éditions du Seuil, 1982.

GRIMAL, Pierre, Dictionnaire de la mythologie grecque et romaine, Paris, Presses universitaires de France, 1951.

HENNARD DUTHEIL DE LA ROCHÈRE, Martine, " "La magie des voix dans la nuit " : “ Transcréation ” des contes de Perrault chez Angela Carter », Études de lettres, 301 (2016), p. 87-108.

-, « Le rire des fées. L'humour dans les contes, de Perrault à Parole », Ricochet-jeunes magazine, 2014.

-, « Jeux de voix chez Perrault et Grimm », Parole, 2 (2015), p. 7-9.

HOOGENBOEZEM, Daphne M., « Magie de l'image : altérité, merveilleux et définition générique dans les contes de Charles Perrault », RELIEF (Revue Electronique de Littérature Française), 4 (2010), p. $1-26$.

JOOSEN, Vanessa et LATHEY, Gillian (eds), Grimms' Tales around the Globe: The Dynamics of their International Reception, Detroit, Wayne State University Press, 2014.

MARIN, Louis, Lectures traversières, Paris, Albin Michel, 1992. 
MONTANDON, Alain (éd.), Iconotextes, Paris, Orphys, 1990.

NIKOLAJEVA, Maria, scotT Carole, How Picturebooks Work, New-York, Routledge, 2006.

NOLDELMAN, Perry, Words about Pictures. The Narrative Art of Children's Picture Books, Athens/London, The University of Georgia Press, 1988.

OPIE, Iona, « Playground Rhymes and the Oral Tradition », in The International Companion Encyclopedia of Children's Literature, ed. by Peter Hunt, London/New-York, Routledge, 1996.

PEYRACHE-LEBORGNE, Dominique (dir.), Vies et métamorphoses des contes de Grimm : traductions, réceptions, adaptations, Rennes, Presses universitaires de Rennes, 2017.

RENONCIAT, Annie (sous la direction de), L'image pour enfants : pratiques, normes, discours (France et pays francophones, $X V I^{e}-X X^{e}$ siècles), Poitiers, La Licorne, 2007.

SERMAIN, Jean-Paul, Métafictions (1670-1730) : la réflexivité dans la littérature d’imagination, Paris, $\mathrm{H}$. Champion, 2002.

-, Le Conte de fées : du classicisme aux Lumières, Paris, Desjonquères, 2005.

SHAVIT, Zohar, Poetics of Children's Literature, Athens/London, The University of Georgia Press, 1986.

TALBOT, Margaret, « The Candy Man : Why Children Love Roald Dahl's Stories-and Many Adults Don't », The New Yorker, 2005 (<http://www.newyorker.com/magazine/2005/07/11/the-candyman>).

VAN DER LINDEN, Sophie, «L'album, entre texte, image et support », La Revue des livres pour enfants, 214 (2003), p 59-68.

-, Lire l'album, Le Puy-en-Velay, l'Atelier du poisson soluble, 2006.

VINAS VALLE, Laura, « Revolting “ Little Red Riding Hood ” ", Didáctica (Lengua y Literatura), 11 (1999), p. 227-250.

WARNER, Marina, Once Upon a Time. A Short History of Fairy Tale, Oxford University Press, 2014.

\section{NOTES}

1. Cet article a été réalisé avec la collaboration de la Prof. Martine Hennard Dutheil de la Rochère (Université de Lausanne), que je remercie ici chaleureusement.

2. Sur la double adresse chez Dahl, voir Z. Shavit, Poetics of Children's Literature.

3. M. Hennard Dutheil de la Rochère, « Le rire des fées », p. 15.

4. M. Nerlich définit l'iconotexte comme «une œuvre dans laquelle l'écriture et l'élément plastique se donnent comme unité insécable» (cité in A. Montandon [éd.], Iconotextes, p. 5 sq.). Dans le même passage, A. Montandon note que «la spécificité de l'iconotexte comme tel est de préserver la distance entre le plastique et le verbal pour, dans une confrontation coruscante, faire jaillir des tensions, une dynamique qui opposent et juxtaposent deux systèmes de signes sans les confondre ".

5. Dans Tales and Translation, p. ix, C. Dollerup propose que les contes de Grimm et Andersen, largement traduits et adaptés depuis le XIXe siècle, fassent désormais partie d'une catégorie plus floue qu'il désigne comme " the international fairytale ».

6. L'histoire éditoriale des contes est indissociable de celle des nursery rhymes en Angleterre. Ainsi, la première traduction anglaise des contes de Perrault par Robert Samber est accompagnée de nursery rhymes dans l'édition de Jacques Barchilon. On rappellera aussi que Alice's Adventures in 
Wonderland, conçu par Lewis Carroll comme un conte (extended fairy tale), fait référence à de nombreux nursery rhymes dont celui de Humpty Dumpty.

7. M. Hennard Dutheil de la Rochère, «Le rire des fées », p. 2.

8. I. Opie souligne que le genre a été le premier à bénéficier d'illustrations dédiées aux enfants dans des ouvrages tels que A Little Book for Little Children (1712) ou Tommy Thumb's Pretty Song Book (1744) (« Playground Rhymes and the Oral Tradition », p. 180).

9. A. Alston et C. Butler (eds), Roald Dahl, p. 24.

10. Les contes de Charles Perrault et surtout ceux des frères Grimm ne reculent devant aucune horreur ou tabou (inceste, infanticide, mutilation, cannibalisme, meurtre, etc.), même si les folkloristes ont exhumé des versions encore plus sanglantes ou choquantes. Comme le résume $\mathrm{M}$. Warner dans Once Upon a Time : «Unspeakable - unbelievable - acts are also always taking place» (p. 79). Le Petit Chaperon rouge chez Perrault se fait dévorer sans que personne ne lui vienne en aide ; chez les Grimm, les demi-sœurs de Cendrillon se font crever les yeux par deux pigeons ; et dans " Blanche-Neige ", la terrible belle-mère est forcée à danser avec des souliers de fer chauffés au rouge, jusqu'à ce que mort s'ensuive.

11. Cette dimension métafictionnelle a notamment été mise en évidence par J.-P. Sermain dans Métafictions.

12. La coquetterie du Chaperon rouge rappelle le conte de Perrault (encouragée par la mère et la grand-mère qui lui fabriquent le vêtement fatal), même si sa cruauté semble plutôt s'inspirer d'un détail troublant dans la version des frères Grimm (c'est elle qui pense à remplir le ventre du loup de pierres).

13. C. d'Humières, D'un conte à l'autre, d'une génération à l'autre, p. 213.

14. L. Viñas Valle, « Revolting “ Little Red Riding Hood ” ", p. 234.

15. S. Beckett, « Perverse, Pistol-Packing Riding Hoods », p. 125.

16. Le Petit Chaperon rouge se comporte comme le héros mythologique qui a tué le lion de Némée pour en revêtir la peau telle une armure invulnérable (cf. P. Grimal, Dictionnaire de la mythologie, p. 191 sq.).

17. S. Beckett, « Perverse, Pistol-Packing Riding Hoods », p. 127.

18. Lettre de Roald Dahl à son éditeur Tom Maschler datée du 21 février 1982 dans laquelle l'auteur exige que d'autres dessins soient réalisés pour The Big Friendly Giant. Archives de l'auteur conservées au Roald Dahl Museum à Great Missenden en Angleterre et consultées du 8 au 12 janvier 2018.

19. Brouillon de James and the Giant Peach, archive consultée le 11 janvier 2018 au Roald Dahl Museum à Great Missenden en Angleterre.

20. Cette démarche se rapproche des stratégies de livres tels que les school primers et rattache donc les récits de Dahl à la littérature éducative.

21. Dans Words about Pictures, Perry Nodelman observe que « [fairy tales] lack the detailed visual description [and] they are sparse in physical detail » (p. 279).

22. M. Nikolajeva, C. Scott, How Picturebooks Work, p. 83.

23. Ibid., p. 49.

24. S. Van Der Linden, Lire l'album, p. 74.

25. Q. Blake, Words and Pictures, p. 58.

26. Ibid., p. 133.

27. A. Renonciat, L'image pour enfants, p. 26.

28. D. Rudd, «Dahl and Language », in Collectif, Roald Dahl, le géant de la littérature jeunesse, p. 63.

29. M. Talbot, « The Candy Man ». Consulté le 27 mars 2016.

30. D. Rudd, « Dahl and Language », Collectif, Roald Dahl, le géant de la littérature jeunesse, p. 65.

31. Collectif, Roald Dahl, le géant de la littérature jeunesse, $p .34$.

32. L. Marin, Lectures traversières, p. 19.

33. M. Hennard Dutheil de la Rochère, « Jeux de voix chez Perrault et Grimm », p. 7. 
34. M. Hennard Dutheil de la Rochère, «Le rire des fées », p. 13.

35. S. Beckett, « Perverse, Pistol-Packing Riding Hoods », p. 128.

36. M. Talbot, « The Candy Man ».

37. Q. Blake, Words and Pictures, p. 82.

38. Dans l'esprit de la version des Grimm, qui optait déjà pour l'avalement tout rond et pour ainsi dire « sans les dents », contrairement au « et il la dévora » de Perrault.

39. Interview de Q. Blake, Lire, p. 39.

\section{RÉSUMÉS}

Roald Dahl et son illustrateur Quentin Blake ont redonné aux contes de fées leur mordant en revisitant de façon à la fois ludique et satirique les contes de Perrault et des Grimm par le texte et l'image à travers le prisme de la tradition anglaise des Mother Goose rhymes. Dahl et Blake, chacun à leur manière, réactualisent la dimension allusive, auto-réflexive et didactique propre au genre afin de proposer une critique drolatique et féroce de la société de consommation et des formes contemporaines de prédation, destinée autant aux enfants qu'aux adultes ${ }^{1}$.

The article shows how Roald Dahl and his illustrator Quentin Blake have restored the biting side of fairy tales through text and image by revisiting Perrault and Grimm's tales in a playful and satirical way through the lens of the British tradition of Mother Goose rhymes. Each in his own way, Dahl and Blake update the allusive, self-reflexive and didactic dimension associated with the fairy-tale genre in order to make fun of consumer society and modern forms of predation addressed to children and adults alike.

\section{AUTEUR}

\section{FLORENCE CASULLI}

Universités d'Angers et de Lausanne 